\title{
Tangence
}

\section{À la source de l'énigme : « Le torrent " d'Anne Hébert}

\section{Janet M. Paterson}

Numéro 50, mars 1996

Lectures de nouvelles québécoises

URI : https://id.erudit.org/iderudit/025889ar

DOI : https://doi.org/10.7202/025889ar

Aller au sommaire du numéro

Éditeur(s)

Tangence

ISSN

0226-9554 (imprimé)

1710-0305 (numérique)

Découvrir la revue

Citer cet article

Paterson, J. M. (1996). À la source de l'énigme : « Le torrent » d'Anne Hébert.

Tangence, (50), 7-19. https://doi.org/10.7202/025889ar d'utilisation que vous pouvez consulter en ligne.

https://apropos.erudit.org/fr/usagers/politique-dutilisation/ 


\section{À la source de l'énigme: "Le torrent " d'Anne Hébert Janet M. Paterson}

[...] la bête a été délivrée. Elle a pris son galop effroyable dans le monde. Malheur à qui s'est trouvé sur son passage.

ANNE HÉBERT, * Le torrent *

Au départ, la situation n'a rien d'inusité ou de particulier. Je choisis d'analyser une nouvelle écrite par une auteure dont je connais de façon intime l'œuvre romanesque. Un seul détail, peut-être, est à noter: la nouvelle en question, *Le torrent ${ }^{1}$, a paru plus de dix ans avant le premier roman d'Anne Hébert et plusieurs décennies avant le reste de l'ouvre qui est toujours en cours de production ${ }^{2}$.

D'emblée, je sais que ma lecture de la nouvelle ne peut être vierge dans le sens où elle est influencée et même filtrée par ma connaissance de l'œuvre entière. Je m'apprête donc à entrer dans un univers fictif dont les contours me seront en grande partie familiers tant par les thèmes que par le style et les structures discursives. À vrai dire, je m'attends même à la présence d'une intèrtextualité interne, sachant de longue expérience qu'elle caractérise l'écriture hébertienne. Mais ce que je n'ai pas anticipé, ce qui va m'interpeller, me poser un défi herméneutique, faire vaciller certaines de mes certitudes, c'est la forme que prendra cette intertextualité et son impact sur l'interprétation de la nouvelle.

1 Anne Hébert, *Le torrent *, Le torrent, Montréal, Éditions $\mathrm{HMH}$, coll. - L'arbre *, vol. 1, 1974, p. 9-65. Toutes les références entre parenthèses renvoient à cette édition.

2 Signalons que la nouvelle - Le torrent - a été publiée, en premier lieu, en 1947, sous le titre .Au bord du torrent , dans Amérique française, vol. VII, $n^{\circ} 2$ (octobre 1947) p. 32-43. Toutefois, la date inscrite à la fin de la nouvelle est: hiver-printemps 1945. Le premier roman de l'auteure, Les chambres de bois, est paru en 1958. 


\section{$\stackrel{* *}{* *}$}

Le déclic aurait pu sans doute se faire de maintes façons puisque, a priori, il dépendait non seulement de ma connaissance des textes, mais de ma sensibilité à des scènes particulières. Comme Leo Spitzer l'a pertinemment noté, un déclic est tributaire d'une multitude de conditions qui varient selon le lecteur ${ }^{3}$. Or, dans mon cas, c'est une phrase particulière, située quelques pages avant la fin du "Torrent", qui a déclenché un processus intense de relecture et de réinterprétation:

La fièvre me glace et me consume. Que fait Amica? [...] Quand elle remontera, je l'étranglerai. Ou plutôt, jattendrai le retour complet de ma vigueur et je jetterai l'espionne dans l'eau. Un instant, mes bras la balanceront au-dessus du précipice. Elle se clébattra. Je ne goûterai pas à ses cris, mais seulement à ses convulsions de terreur. Puis, Amica sera décapitée et démembrée. Ses débris bondiront sur les rochers. (p. 61)

Aucun concept critique, c'est-à-dire ni intertextualité, ni palimpsesté et encore moins allusion, ne peut tenir compte de mon expérience herméneutique. Dans l'espace de ces quelques phrases, ma compréhension de la nouvelle entière est transformée. Un voile est abruptement tiré alors que le monde sinistre et violent des Fous de Bassan 4 s'impose de façon implacable et décisive. Évidemment, je me mets à la relecture du "Torrent ", qui n'est plus, qui ne peut plus être le texte que j'ai connu dans ses fonctionnements formels et signifiants tant il est modifié par le roman, publié pourtant presque trente-cinq ans après la parution de la nouvelle. Comment en effet ne pas faire le lien entre les deux textes à partir de cette scène fantasmée d'étranglement et de noyade, scène où la référence aux cris déclenche, avec une clameur retentissante, l'écho des cris surgissant des Fous de Bassan? Comment ne pas entendre, dans l'interstice des mots, les cris des oiseaux entremêlés à ceux des personnages, cris assourdissants qui expriment la douleureuse impasse du désir et la béance de son inassouvissement?

Lorsque, face à ces homologies, je compare la nouvelle au roman, j'aperçois un processus de superposition dynamique qui

3 Leo Spitzer, Linguistics and Literary History: Essays in Stylistics, Princeton, Princeton University Press, 1948.

4 Anne Hébert, Les fous de Bassan, Paris, Seuil, 1982. 
engendre la cohabitation d'un texte par l'autre. En effet, l'univers entier du "Torrent" est radicalement transformé par celui des Fous de Bassan, alors que ce dernier est hanté par les réseaux obsessionnels du premier. Au départ, cette cohabitation m'apparaît dans un mélange désordonné d'éléments hétérogènes - personnages dédoublés, cris stridents, eau déchaînée, corps démembrés - éléments que je m'efforce d'isoler pour en sonder les formes et les sens.

\section{Les récits en miroir}

Sur le gros plan que forment les deux textes se dégagent, de façon magistrale, les figures des personnages principaux, François et Stevens. Ainsi placés côte à côte, ils accusent des ressemblances tout à fait étonnantes, et ce, au niveau-même du discours. Faire le repérage de ces ressemblances s'avère une véritable excavation dans la mesure où chaque couche homologique, une fois découverte, en révèle une autre encore plus signifiante et souvent plus inattendue. Comparant l'incipit des deux textes, je remarque que François et Steven sont tous les deux énonciateurs, c'est-à-dire maîtres d'une parole qui se manifeste sous le signe du " je " narratif, dès la première inscription textuelle. "J'étais un enfant dépossédé du monde" (p. 9), s'exclame François au début de la nouvelle alors que le récit de Stevens, écrit sous forme de lettres, commence ainsi : Me voici donc de retour au pays natal" (p. 57). Est-il nécessaire de souligner la mise en relief inaugurale du sujet de la narration (le *j'*, le *me*) dans les deux textes? Faut-il insister sur le mouvement, tout aussi immédiat, vers le passé, d'un côté par l'emploi de l'imparfait, "j'étais ", renforcé par le mot *enfant *, et de l'autre, par l'usage des mots * retour" et "natal"? Et enfin doit-on faire remarquer que, dans les deux discours, le sujet énonciatif se situe d'emblée en relation à l'espace physique qui l'entoure, à savoir le "monde " et le "pays"? Se faisant écho de manière fulgurante, ces germes de sens dévoilent la présence de structures formelles dont l'importance est primordiale autant pour la production du sens dans chaque texte que pour leur relation réciproque.

L'incipit du "Torrent" met en marche, en effet, tout le déroulement du texte. Il s'agit d'un discours narré à la première personne, où le protagoniste-narrateur, François Perrault, âgé d'une quarantaine d'années, pose un regard rétrospectif sur son passé. 
Son récit se divise en deux parties. La première, écrite au passé, raconte les tristes détails de son enfance et de son adolescence, alors que la deuxième, narrée au présent, fait état de son expérience adulte. Or, c'est exactement la même structure que l'on retrouve dans Les fous de Bassan. Le personnage narrateur, Stevens Brown, âgé de vingt ans, raconte les péripéties de son retour à Griffin Creek et ses souvenirs d'enfance et d'adolescence. Quarante-six ans plus tard, il revoit, dans un deuxième récit, certains moments cruciaux de sa vie adulte.

Mais quelle est la finalité, peut-on se demander, de ces regards insistants vers le passé? En retraçant patiemment des trajectoires vécues, qu'est-ce que les narrateurs espèrent? Qu'est-ce qu'ils réclament? On s'aperçoit que, dans les deux cas, il s'agit d'une interrogation et d'une quête. Ce n'est sûrement pas par hasard qu'en relatant son passé, François fait allusion à un "manque "qu'il se "harcèle à éclaircir" (p. 35) par le truchement d'une *investigation" "lucide et méthodique. (p. 38). Manque qui tourne, comme on le sait, autour de la mort brutale de sa mère. Quant à Stevens, s'il ressasse des souvenirs d'enfance - "tentative effrénée et vaine de compréhension filiale. (p. 87) - c'est aussi dans le but de mettre en lumière un manque, en l'occurrence l'énigme des crimes commis la nuit du 31 août 1936.

Pourtant, dans un récit comme dans l'autre, il s'agit moins d'une histoire de meurtres (lesquels sont d'ailleurs curieusement absents des deux diégèses) que de l'exploration de l'aliénation ou, comme le dit François, de la "dépossession" qui est à la source d'une violence impitoyable.

\section{Le retour vers le passé}

Dire la dépossession, en cerner l'origine, en examiner la douleureuse évolution, entraîne inévitablement un retour vers l'enfance. À ce niveau, aucun commentaire critique ne peut rendre adéquatement compte de l'ampleur de la brutalité et de la destruction qui caractérisent le rapport de la mère de François, la grande Claudine, à son fils. Rapport sadique, rapport dénaturé, où les privations et la violence physique et morale se multiplient. Pour tenter d'en faire état, on pourrait certainement rappeler que Claudine bat son fils régulièrement jusqu'à le rendre sourd lors d'un moment de confrontation; ou encore, on pourrait signaler qu'elle le prive complètement d'affection, lui interdisant même le contact 
avec autrui. Mais en fin de compte, c'est le discours de François, fruit amer de sa lucidité, qui met en pleine évidence l'étendue du pouvoir castrateur de la mère: "Ô ma mère, que je vous hais! et je n'ai pas encore tout exploré le champ de votre dévastation en moi. " (p. 56); "Ah! ma mère, je ne pouvais deviner toute l'ampleur de votre destruction en moi!. (p. 49); "Ces révélations m'atteignaient douleureusement. En une seconde, je mesurais le néant de mon existence. Je pressentais le désespoir " (p. 24).

"Dévastation ", "destruction ", "néant " et "désespoir": ces mots s'agglutinent, se font écho, se répètent pour ancrer de façon inexorable les signes de la dépossession dans le discours, alors même que le "je", le "moi", prévoit sa destruction par le "vous" maternel ${ }^{5}$.

Parallèlement, si le récit de Stevens s'amorce par la notion de retour, c'est pour effectuer un mouvement discursif vers des scènes dévastatrices de l'enfance. À certains moments, on a l'impression que les deux récits convergent, se fondent l'un dans l'autre, tant les scènes de violence physique et de manque affectif se ressemblent. Il y a pourtant une différence qui se dégage des Fous de Bassan. Dans son enfance, comme dans son adolescence, Stevens est maltraîté à la fois par sa mère et son père: *Ce froid vient d'ailleurs, des profondeurs confuses de la naissance, du premier attouchement des mains glacées de ma mère sur mon corps d'enfant" (p. 86); "Ce n'est pas le lait tout cru qu'elle m'a donné, Béatrice ma mère, c'est la faim et la soif. Le désir. Je dois hurler aussi fort que Perceval pour que mon père me chasse, à coups de pied, de la chambre froide. (p. 87); "John Brown doit avoir de bonnes raisons pour cogner si fort sur la tête de son fils, sur le dos de son fils, sur les fesses de son fils, dès que l'occasion se présente. (p. 87).

De la privation et de la brutalité à la dépossession émotive et spirituelle de l'être humain, il n'y a qu'un pas, comme le révèlent les deux récits. Figures doubles d'une même psyché ravagée, François et Stevens sont tous les deux violents et colériques. Se nourrissant de fantasmes d'étranglement et de noyade, ils deviennent les prototypes du meurtrier. D'où l'importance diégétique,

5 Pour une étude de la dépossession perçue en termes d'exil spatial et psychologique, voir Neil B. Bishop, Anne Hébert, son cutre, leurs exils, Bordeaux, Presses Universitaires de Bordeaux, 1993, en particulier, les chapitres V et VI. 
dans les deux textes, du motif du meurtre et des références à la police et au verdict du coroner ${ }^{6}$. Mais afin d'explorer plus profondément cet aspect de l'ouvre, il faut s'attarder à une autre figure qui émerge de l'entre-deux des personnages, qui s'impose avec insistance sur l'écran visuel des textes.

\section{Perceval : cet autre moi-même}

S'il y a convergence entre François et Stevens, de telle sorte qu'ils forment à certains moments une seule et même personne, leur figure double est envahie, dans les deux textes, par l'ombre d'un "personnage "nommé Perceval. Que dire de cette redondance onomastique, de cette reprise d'un prénom déjà intertextuellement motivé ${ }^{7}$, sinon qu'elle nous incite, encore une fois, à rapprocher les récits.

Dans "Le torrent", Perceval est un cheval, quasi sauvage, qui surgit mystérieusement un jour chez François. Tout noir, il se caractérise tant par son audace et sa persévérance que par son déchaînement et sa "rage étonnante" (p. 34). Véritable "démon captif "( p. 34) aux "naseaux fumants "et à "l'écume sur le corps" (p. 31), il doit combattre Claudine qui, en dépit de l'état sauvage de la bête, l'enferme dans la grange. Plusieurs détails dans le texte révèlent le caractère indigne de cet emprisonnement: "le sang sur son poil se mêlait à la sueur. Il était cruellement entravé ", "superbe créature blessée et torturée" (p. 34). Meurtri, le cheval devient littéralement enragé : - Le cheval se démenait si fort que je craignais qu'il ne défonçât tout* (p. 34). Enragée, la bête l'est jusqu'à ce qu'elle reprenne * son galop effroyable dans le monde" (p. 36), et ce, par-dessus le corps gisant de Claudine.

6 Il faut en effet souligner que les références à la police et à l'interrogatoire, si évidentes dans Les fous de Bassan, se manifestent également dans * Le torrent ": "Cela me fait penser au chef de police, à l'interrogatoire qu'il me fit subir après la mort de ma mère!- (p. 59); "J'ai devant les yeux le verdict du coroner: "Mort accidentelle". Qu'a donc cette fille à perquisitionner ici?. (p. 61).

7 Pour une étude du rappórt intertextuel de la nouvelle au roman de Chrétien de Troyes, Le roman de Perceval ou le conte du Graal, voir mon article *Ah! Perceval, qui étiez-vous donc?", Solitude mompue, textes réunis par Cécile Cloutier-Wojciechowska et Réjean Robidoux en hommage à David M. Hayne, Ottawa, Éditions de l'Université d'Ottawa, 1986, p. 287-293. Voir également Geneviève Ceschi, . À la source du Torrent d'Anne Hébert •, thèse de mầtrise, Université de la Colombie-britannique, 1978, f. 40-72. 
Dans Les fous de Bassan, par contre, Perceval prend une forme humaine: le prénom désigne un adolescent faible d'esprit qui est le jeune frère de Stevens Brown. L'importance de son rôle dans le roman est soulignée à deux niveaux: à celui du discours, car à l'instar de Stevens, Perceval prend la parole còmme énonciateur dans un des récits, et à celui de l'intrigue, puisqu'il remplit la fonction de témoin lors des meurtres. Rien, donc, à première vue ne signale une parenté avec le cheval, à moins que l'on se penche sur des aspects particuliers du personnage. Ce faisant, on s'aperçoit que ce n'est pas par hasard si le jeune Perceval porte le nom du cheval. Comme lui, il est fréquemment enfermé contre son gré : "Enfermé tous les soirs dans la maison [...] Enfermé dans ma chambre pour la nuit [...] Envie de crier. Parce que je suis enfermé. (p. 139); comme s'il s'agissait d'un cheval, il est souvent battu, "serai battu si je crie" (p. 139), "ma grand-mère a tout raconté et mon oncle John a battu Perceval avec un fouet comme on bat un cheval. (p. 117); comme la bête, enfin, Perceval est en proie à des colères et à des rages: "J'en viens au hurlement pur, sans mots distincts" "cri informe, profond de la bête qui appelle" (p. 151). Perceval serait-il une bête? Dans le contexte d'une homologie avec le cheval frémissant du *Torrent", le glissement devient effectivement opératoire. Mi-personnage, mi-bête, Perceval finit par incarner l'animal sauvage avec lequel il se confond de façon tout à fait saisissante: "Il est plein de vapeur blanche, comme un train en marche, un cheval au galop les naseaux fumants, du moins il le croit et court, de plus en plus fort" (p. 83):

Constater la présence d'une superposition entre le cheval du "Torrent "et l'adolescent des Fous de Bassan est toutefois important à un autre niveau, car cette superposition entraîne un brouillage actantiel lourd de conséquences. Dans les deux récits, l'image de Perceval s'agglutine étroitement à celle du personnage principal. Comme Robert Harvey l'a pertinemment démontré ${ }^{8}$, le cheval dans "Le torrent" est une incarnation de la passion de François. En s'opposant à la gigantesque Claudine et en laissant libre expression à sa rage et à sa colère, il figure l'être de "fougue et de passion" (p. 31) que François aimerait représenter. De

8 Robert Harvey, Kamouraska d'Anne Hébert: Une écriture de la passion suivi de Pour un nouteau Torrent, Montréal, Hurtubise HMH, 1982, p. 129-184. Voir surtout p. 136-138. 
14

même, dans Les fous de Bassan, Perceval constitue, de l'aveu de Stevens, son double, son "autre moi-même" (p. 249). Or, comme Marilyn Randall l'a bien mis en évidence, ce processus est réciproque :

Par le truchement du dédoublement entre Perceval et Stevens, il semble s'opérer un échange d'attributs. Si la folie de Perceval extériorise l'alter ego submergé et refoulé de Stevens, Perceval ne serait-il pas aussi représentatif de l'innocence sauvage et déchaînée de Stevens?"

Tributaire de ces dédoublements, une nouvelle figure, quasi mythique, se dégage des deux récits. Figure où se confondent dans une seule et même personne, non seulement François et Stevens ou bien François et Perceval, ou encore Stevens et Perceval, mais davantage, les quatre personnages ensemble. Figure puissante qui, en plus d'imbriquer de façon irrévocable la nouvelle dans le roman, pose la question énigmatique de l'identité du meurtrier, question à laquelle je reviendrai plus loin.

\section{“L'eau est noire [...] et l'écume crache jaune »}

Dans la fresque qui émerge de plus en plus vivement des deux récits, se font entendre, par l'entremise même des personnages, le grondement du torrent et le hurlement des vagues de la mer. À tel point qu'on peut se demander si ces eaux proviennent d'un même lieu discursif et symbolique ${ }^{10}$.

Pour répondre à cette question, examinons tout d'abord la présence diégétique du torrent. Curieusement, au niveau de l'espace, le torrent se manifeste dans le récit seulement lorsque François devient sourd. Avant cet incident, la campagne, les champs, les ruisseaux, la route et le village sont évoqués, mais jamais le torrent. Or, à la différence de ces autres éléments spatiaux, l'inscription du torrent dans le texte se caractérise immédiatement par un processus de symbolisation. Sous la pulsion d'une

9 Marilyn Randall, - Les énigmes des Fous de Bassan: féminisme, narration et clôture , Voix et images, $\mathrm{n}^{\circ} 43$ (vol. XV, $\mathrm{n}^{\circ} 1$ ), automne 1989), p. 80.

10 Pour des études détaillées du symbole du torrent voir Pierre-Hervé Lemieux; "La symbolique du "Torrent" d'Anne Hébert", Revue de l'Université d'Ottawa, vol. LXIII, $\mathrm{n}^{\circ}$ 1, janvier-mars 1973, p. 114-127; Lucille Roy-Hewitson, "Anne Hébert "Le Torrent" ou l'intégration au cosmos", The French Review, $\mathrm{n}^{\circ} 53,1980$, p. 826-833. 
forte surdétermination, le "rythme précipité de l'eau houleuse" et son "tumulte intermittent" (p. 30) expriment l'angoisse et le bouillement intérieurs du personnage:

Mais, les jours épouvantables où je ressassais ma révolte, je percevais le torrent si fort à l'intérieur de mon crâne, contre mon cerveau, que ma mère me frappant avec son trousseau de clefs ne m'avait pas fait plus mal. (p. 30)

Il faut remarquer toutefois qu'une progression marque le déploiement du symbole, car le tumulte s'avère de plus en plus violent et sauvage. Tout d'abord, il donne lieu à un spectacle brutal de "plusieurs luttes exaspérées, de plusieurs courants et remous intérieurs se combattant férocement. (p. 32). Ensuite, lorsque le "vacarme "et le "fracas" (p. 32) du torrent deviennent insupportables pour François, ce sont des images de corps démembrés qui surgissent des profondeurs de la cascade: "Le rocher était limoneux. C'eût été facile de glisser. Quel saut de plusieurs centaines de pieds. Quelle pâture pour le gouffre qui devait décapiter et démembrer ses proies! Les déchiqueter..." (p. 33).

Doit-on s'étonner que la prochaine scène soit celle du déchaînement du cheval et de la mort de la mère? Ou encore, est-il surprenant que cette mort, dont la description est absente du discours, soit signifiée par l'entremise du torrent: "Le torrent subitement gronda avec tant de force sous mon crâne que l'épouvante me saisit" (p. 35); "Le torrent me subjugua, me secoua de la tête aux pieds, me brisa dans un remous qui faillit me désarticuler* (p. 35). Symbole d'une force sauvage, dont la parenté avec le cheval est maintes fois signalée dans le texte ${ }^{11}$, le torrent incarne l'expression du désespoir et de la rage meurtrière de François.

Dans la deuxième partie du récit, narrée à peu près vingt ans plus tard, le torrent se manifeste de nouveau selon des mouvements divers. Au départ, il est silencieux: a Le torrent est silencieux. Du silence lourd qui précède la crue du printemps" (p. 38). Mais presque immédiatement après la rencontre avec Amica, François sent "le murmure lointain du torrent, en marche" (p. 48) vers lui. Et lorsque ses sentiments pour Amica se transforment en méfiance et en peur ( Cette fille [...] m'observe et m'épie ",

11 Voir Robert Harvey, op. cit., p. 138. 
16

p. 56), le torrent redevient subitement actif et violent: "Ah! qui me frappe avec cet acharnement? Le torrent bondit dans ma tête!" (p. 56). Comme dans la première partie du texte, des fantasmes d'étranglement et de démembrement s'emparent de François : "Quand elle remontera, je l'étranglerai [...] Puis, Amica sera décapitée et démembrée" (p. 61). À la fin du récit, tout se passe comme si le déchaînement du torrent était incontrôlable. Issu de forces violentes, il envahit l'univers entier du narrateur le souvenir d'Amica, la maison de l'enfance, le visage de la mère - jusqu'à ce que, dans un dernier geste, sa puissance engouffre l'existence même du personnage, tout en axant le discours sur l'ultime aventure de l'écriture: "Je me penche tant que je peux. Je veux voir le gouffre, le plus près possible. Je veux me perdre en mon aventure, ma seule et épouvantable richesse" (p. 65).

\section{«Des vagues de dix pieds de haut, des crêtes d'écume »}

Déplaçant mon regard du *Torrent", je scrute maintenant la mer dans Les fous de Bassan, afin de voir si elle est gouvernée par le même processus signifiant. D'emblée, je suis frappée par une homologie diégétique. Élément spatial de prime abord dans le récit de Stevens, tant à Griffin Creek qu'à Key West, la mer assume une valeur symbolique à la suite d'un incident particulier. C'est en effet après la visite de Stevens chez ses parents (visite d'ailleurs plusieurs fois remise), pendant laquelle il est hanté par le souvenir de leur violence à son égard, que la mer est dotée de traits négatifs :

Dans cette eau qui moutonne, dont chaque vague moutonne et crépite, pareille à des balles de fusil, mille balles de fusil lâchées ensemble, une muraille crépitante qui se forme, monte, atteint son sommet, s'affaisse aussitôt, écumante sur le sable, mourante sur le sable. (p. 95)

Dans ce passage, la mer est soumise à un processus de surdétermination maximale: la répétition de l'image des uballes de fusil "se rattache à celle de la muraille "mourante sur le sable " pour exprimer la violence et le désir refoulés de Stevens. On pourrait même affirmer qu'en réalité, le discours devance ici l'intrigue, puisque le prochain incident représente justement une scène de violence sexuelle. Errant sur la grève, Stevens y rencontre Olivia et tente de l'embrasser. Sujet à des passions violentes, il 
s'empare de l'adolescente de façon assez brutale jusqu'à ce que ses frères interviennent. Dès lors, frustré, enragé, il exprime sa colère par l'entremise de la grande tempête qui agite la mer: "La mer déchaînée déferle sur la grève" (p. 102).

C'est toutefois dans la dernière lettre de Stevens que la mer assume la plénitude de son pouvoir symbolique, lors de la confrontation avec Nora (confrontation qui n'est pas sans rappeler la scène pendant laquelle François se sent menacé par Amica): "à mesure que Nora m'injuriait et m'insultait [...] le coup de vent s'est levé sur la mer [...] S'engouffre à nouveau dans ma tête le fracas de la haute mer en marche" (p. 244-245). Et enfin, dans un mouvement qui relie inextricablement "Le torrent" aux Fous de Bassan, Stevens, comme François - c'est-à-dire en utilisant les mêmes motifs et les mêmes symboles - décrit des scènes d'étranglement dans son récit qui s'écrit sur une "sorte de page blanche" (p. 233): "Le cri sous mes doigts, dans sa gorge [...] Très vite Olivia rejoint Nora à mes pieds, sur le sable de Griffin Creek [...] j'emmène mes cousines au large, alourdies de pierres et de cordes" (p. 248-249).

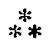

De cette lecture, qui s'est penchée sur l'entrelacement de deux textes hébertiens, ressortent plusieurs considérations, la première ayant trait à la notion de meurtrier innocent. Dans son article quelque peu controversé, Marilyn Randall a avancé que Les fous de Bassan est un roman profondément énigmatique, en particulier à cause du dénouement de l'intrigue: "Roman policier par excellence, il se termine sur une confession du criminel qui laisse pourtant perplexe" ${ }^{12}$. À son avis, Stevens n'est pas nécessairement le meurtrier de Nora et d'Olivia, en dépit de son aveu à cet effet (aveu d'ailleurs généralement accepté par les critiques). Si l'on tient compte du dédoublement entre Stevens et François et de la superposition d'un texte à l'autre, on s'aperçoit qu'effectivement la description du meurtre de Nora et d'Olivia par Stevens, comme celle de François, pourrait vraisemblablement provenir d'un fantasme. Plusieurs détails appuient cette hypothèse. On 
sait, par exemple; que le bruit du torrent, maintes fois décrit par François, ne peut qu'être imaginaire puisque le personnage est sourd. De même, ou bien Stevens ment, ou bien il succombe au fantasme total quand il décrit la fureur de la mer, lors de la nuit du crime, étant donné que, selon tous les villageois, l'océan était parfaitement calme cette nuit-là. Ainsi, dans les deux récits, - l'aveu " du narrateur s'inscrit, non sans conséquences, dans un cadre fantasmé.

Outre l'homologie frappante entre les deux scènes de meurtre, l'incident de la mort de Claudine confirmerait l'innocence de Stevens, tout en apportant un éclairage supplémentaire à l'intrigue. Rappelons que c'est au comble de la rage de François que le cheval Perceval, dans un désir fou de libération, piétine Claudine à mort. Qu'est-ce à dire? Que, dans Les fous de Bassan, c'est également Perceval, le frère de Stevens, qui aurait tué Nora et Olivia? Et que son frère en aurait pris la responsabilité? Comme l'a signalé Randall, il est fort possible que Stevens ait voulu protéger la seule personne au monde qu'il a "jamais aiméle]" (p. 249), sans rien dire des pistes discursives qui corroborent une telle lecture ${ }^{13}$. Dans ce cas, le soi-disant aveu de Stevens ne s'éloignerait pas tellement du récit fantasmé de François où percent si fréquemment des sentiments de culpabilité. Perceval serait donc, dans les deux récits, le meurtrier? Rien n'est moins sûr. Ce que la juxtaposition des textes nous permet d'entrevoir, c'est précisément que, chercher, à l'instar des policiers dans "Le torrent" et dans Les fous de Bassan, le vrai meurtrier, le seul coupable, c'est passer à côté de l'essentiel. En mélangeant l'expression du désir violent et de sa réalisation, ces textes mettent en évidence la présence non pas d'un seul, mais de plusieurs meurtriers. Ce n'est pas en effet pour rien que les discours passent si souvent du littéral au figuré: en brouillant les frontières entre le fantasme et le réel, ils rattachent la violence meurtrière à plusieurs personnages - François, Perceval, Nicolas, Perceval Brown, Stevens et Bob Allen. Qui plus est, derrière leurs figures déchirées surgit souvent l'ombre de parents maléfiques, eux-mêmes meurtriers ${ }^{14}$. Ce qui apparaît ainsi dans

13 Ibid., p. 79-82. Randall analyse en détail les indices textuels qui contredisent, ou qui, du moins, mettent en doute l'aveu final de stevens.

14 Voir, par exemple, dans Les fous de Basian, les paroles de Stevens: - Mon père dévale la pente du sentier et s'abat sur moi pour me tuer. Ma mère est d'accord pour qu'il me tue. (p. 239); dans - Le torrent ", la mort du cheval Eloi, clont Claudine est responsable, suggère la puissance meurtrière de celle-ci. 
ces récits, ce qui nous interpelle, n'est pas la question de l'identité des meurtriers, mais plutôt celle du drame de la dépossession et de ses conséquences néfastes: "La bête a été délivrée. Elle a pris son galop effroyable dans le monde. Malheur à qui s'est trouvé sur son passage" (Le torrent", p. 36). C'est ainsi, qu'en dernière analyse, la réponse à l'énigme des Fous de Bassan se trouve dans "Le torrent".

$\mathrm{Si}$, pour conclure, la lecture du "Torrent" éclaire le mystère des Fous de Bassan alors que ce dernier modifie notre perception de la nouvelle, force nous est de reconnaitre la puissance matricielle du récit célèbre d'Anne Hébert. Puissance qui se manifeste non seulement au sein du recueil, comme l'a pertinemment démontré Jean-Pierre Boucher ${ }^{15}$, mais également dans l'ensemble des textes hébertiens, ainsi que l'a constaté Harvey ${ }^{16}$. Dans le cas des Fous de Bassan, toutefois, le pouvoir générateur de la nouvelle prend des proportions inédites dans la mesure où il établit une relation symbiotique entre deux textes publiés à trente-cinq ans d'intervalle. Relation où pointe, à travers le fracas du torrent et la fureur de la mer, dans le déchaînement du désir, le sens de ce qu'on appelle une œuvre littéraire.

15 Jean-Pierre Boucher, - Nouvelle éponyme en ouverture: Le Torrent d'Anne Hébert, Le recuell de nouvelles. Études sur un genre littéraire dit mineur, Montréal, Fides, 1992, p. 25-37.

16 Robert Harvey, op. cit. L'étude de Harvey inclut les textes hébertiens publiés avant 1982. 